\title{
The EC-system of EU DEMO: concepts for a reactor heating system
}

\author{
G. Granucci ${ }^{1}$, G. Aiello ${ }^{2 b}$, K.A. Avramidis ${ }^{2 \mathrm{a}}$, A. Bruschi ${ }^{1}$, G. Gantenbein ${ }^{2 \mathrm{a}}$, \\ S. Garavaglia ${ }^{1}$, G. Grossetti $^{2 b}$, J. Jelonnek ${ }^{2 \mathrm{a}}$, A. Moro ${ }^{1}$, E. Poli ${ }^{5}$, N. Rispoli ${ }^{1}$, D. Strauss ${ }^{2 \mathrm{~b}}$, M. Thumm ${ }^{2 \mathrm{a}}$, \\ I. Tigelis ${ }^{4}$, C. Tsironis ${ }^{4}$, T. Franke ${ }^{5,6}$ and M.Q. Tran ${ }^{3}$ \\ ${ }^{1}$ Institute of Plasma Physics "P.Caldirola", National Research Council of Italy, Milan, Italy \\ ${ }^{2 \mathrm{a}} \mathrm{IHM},{ }^{2 \mathrm{~b}}$ IAM-AWP, Karlsruhe Institute of Technology (KIT), D-76131 Karlsruhe, Germany \\ ${ }^{3}$ Swiss Plasma Center, EPFL, CH-1015 Lausanne, Switzerland \\ ${ }^{4}$ NCSRD/National and Kapodistrian University of Athens, Faculty of Physics, \& National Technical University of Athens, \\ School of Electrical and Computer Engineering, Athens, (Greece) \\ ${ }^{5}$ Max-Planck-Institut für Plasmaphysik, Boltzmannstr. 2, D-85748 Garching, Germany \\ ${ }^{6}$ EUROfusion Consortium, Boltzmannstr. 2, D-85748 Garching, Germany
}

In the frame of the project for a European DEMOnstration Fusion Power Plant (FPP), the design of the auxiliary heating systems is one of the keys to heat and control the burning plasma (i.e. a plasma in reactor producing electricity to the grid from fusion reactions). The heating mix considered in EU DEMO bases on Electron Cyclotron Resonance Heating and Current Drive (ECRH\&CD), Neutral Beam Injection (NBI) and Ion Cyclotron Resonance Heating (ICRH). In this first phase of the design process (called Pre-Conceptual phase) each system is under study with $50 \mathrm{MW}$ as a reference power to the plasma [1]. The main tasks assigned to the EC system are reported in the Table 1, assuming the full use of the $50 \mathrm{MW}$ :

Table 1. Main Physical Requirements for EC system

\begin{tabular}{|l|c|c|c|}
\hline \multicolumn{1}{|c|}{ EC Task } & $\begin{array}{c}\text { Power } \\
{[\mathbf{M W}]}\end{array}$ & $\begin{array}{c}\text { Localiza- } \\
\text { tion }[\rho]\end{array}$ & Mode \\
\hline $\begin{array}{l}\text { Assisted Break- } \\
\text { down }\end{array}$ & $6-10$ & $<0.3$ & Heating \\
\hline $\begin{array}{l}\text { Ramp up and } \\
\text { L-H transition }\end{array}$ & 50 & $<0.3$ & Heating/CD \\
\hline Main heating & 50 & $<0.3$ & Heating/CD \\
\hline Sawtooth control & $2-10$ & $<0.3$ & CD \\
\hline $\begin{array}{l}\text { NTM control } \\
(\mathrm{q}=2 ; \mathrm{q}=3 / 2)\end{array}$ & $10-15$ & $0.85 ; 0.75$ & $\mathrm{CD}$ \\
\hline Ramp down & 40 & $0.3-0.5$ & Heating \\
\hline
\end{tabular}

These heating tasks require different radial localization and combination of heating and non-inductive current drive (CD). A possible solution is a gyrotron capable to operate at two (or more) different frequencies, in order to cover all the physical requirements in the easiest way. Moreover in designing an EC system for DEMO, it is necessary to consider the peculiarities of a fusion power plant reactor, which are significantly different from those of any existing tokamak experiments. The power reliability and availability are major targets for the system, because in case of fault of EC system the pulse must be terminated, with a consequent unacceptable economic impact. Therefore, reliability of any each sub-system is taken into consideration when choosing the architecture of the EC system. A second objective is to minimize the recirculating power to guarantee the higher possible energy gain of the reactor with the use of a highly efficient gyrotrons [2] (above $60 \%$ ) and low losses (10\%) transmission lines.

The present design considers $2 \mathrm{MW}$ gyrotron units, capable to operate at $170 \mathrm{GHz}$ for heating and $204 \mathrm{GHz}$ for current drive. This last corresponds to a moderate upshift of 1.2 for the CD function while the two frequencies $(170 / 204 \mathrm{GHz})$ have been selected to satisfy the $\lambda / 2$-resonances of the diamond-disc window. These frequencies have been selected for the pulsed EU DEMO1 2015 baseline [3] and used as reference to calculate CD efficiency and localization. At the end of the EU DEMO Conceptual Design phase, the appropriate frequencies will be selected. Within EUROfusion research is ongoing to consider gyrotron operating frequencies of up to $240 \mathrm{GHz}$.

The transmission line (TL) is based on a quasioptical approach, inspired to the Multi-beam (MB)TL of W7-X stellarator experiment [4], enclosing the mirrors in an evacuated pipe to make it compatible with the nuclear environment. The concept is based on a mirror confocal layout with single units composed by two mirrors forming a dog-leg and replicated few times for transmitting along straight paths (150 $\mathrm{m}$ as reference length).

Table 2. Transmission losses estimation of DEMO EQO for a length of $150 \mathrm{~m}$

\begin{tabular}{|l|c|c|}
\hline Distance between mirrors $=8 \mathrm{~m}$ & $170 \mathrm{GHz}$ & $204 \mathrm{GHz}$ \\
\hline Ohmic Losses (H-plane - 45 $)$ & $3.2 \%$ & $3.5 \%$ \\
\hline Truncation (40 mirrors) & $1.5 \%$ & $1.4 \%$ \\
\hline Mode conversion & $1.5 \%$ & $1.4 \%$ \\
\hline Total Losses & $6.2 \%$ & $6.3 \%$ \\
\hline
\end{tabular}

One pumping system is located in each mirror unit. As reference it is considered to transmit 8 beams with one MBTL, with an overall losses estimated to be $<10 \%$ (see Table 2). This choice would allow a simpler and compact solution capable to reduce the complexity of the system and to save space.

The design of the launching system is strongly limited by severe constrains imposed by the environment of a nuclear reactor as the vicinity of a hot burning plasma and under a heavy neutron bombardment. In particular, no movable parts are allowed in proximity of a burning plasma, and shielding and suitable structural component need to be included in the design [5]. A remote steering antenna, with a dogleg in the vessel port, is under study as one of the options for a launcher concept. The proper choice of steering plane and the inclination of the corrugated square waveguide are the free parameters for the required EC power localization and CD efficiency, to be combined with the engineering requirements. In Fig. 1 the result of a beam tracing performed with TORBEAM [6] is shown. Using both the frequencies the possibility to cover the assigned tasks of Table 1 is demonstrated, 
while the $204 \mathrm{GHz}$ exhibits a higher central CD efficiency (for ST control), the $170 \mathrm{GHz}$ can be efficiently used for NTM control and for central or off-axis heating.



Fig. 1. With full lines (dashed lines) calculated CD profiles for $170 \mathrm{GHz}(204 \mathrm{GHz})$ launched from equatorial plane with antenna toroidally inclined of of $17^{\circ}\left(22^{\circ}\right)$. For the different depositions a steering of $\pm 12^{\circ}$ is considered

A comparison of remote steering antennas and alternative simpler solutions, truncated waveguides used as launchers with step tunable gyrotron, is ongoing, on the basis of the physical requirements of Table 1 . This study can be complemented considering the use of step tunable gyrotrons used in the truncated waveguide launcher concept, in order to have enough localization control for NTM stabilization. The other tasks can be satisfied increasing the number of launching points with the aid of fast microwave switches.

The reliability of the auxiliary power to be delivered, and, more in general, the RAMI of the whole EC system, is another characterizing point of the conceptual design. The maximization of reliability is obtained with appropriate system architecture, characterized by a modular design. An ideal overall reliability of $100 \%$ to deliver $50 \mathrm{MW}$ to the DEMO plasma using an EC system is possible to reach by considering a proper redundancy in the different sub units. Therefore, the system concept is based on a strong modularity which considers a cluster of 8 sources (gyrotrons) fed by one high voltage cathode power supply and individual anode power supply. The 8 beams are transmitted to the launchers with a single MBTL. The launcher of each cluster is individual and in the same port plug in structure. The best combination [7] is composed by 5 clusters of 8 units, with 7 gyrotrons operating in each cluster (and one in standby) and one full cluster in standby. Therefore only 28 lines will be on simultaneously with 12 lines off ready to substitute the unit (gyrotron or line / launcher) eventually in fault.

\section{Acknowledgement}

Parts of this work are carried out within the framework of the EUROfusion Consortium and have received funding from the Euratom research and training programme 2014-2018 under grant agreement No. 633053. The views and opinions expressed herein do not necessarily reflect those of the European Commission.

\section{References}

1. M. Q. Tran et al. D. EU DEMO Heating and Current Drive: Physics and Technology // 26th FEC - Kyoto (2016) FIP/7-7.

2. J.Jelonek et al European Research Activities Towards a Future DEMO Gyrotron // SMP, 2017, Nhizhny Novgorod.

3. R. Wenninger et al. The physics and technology basis entering European system code studies for DEMO // Nucl. Fusion 57, 016011 (2017).

4. V. Erckmann et al. Electron cyclotron heating for W7-X: physics and technology // Fusion Sci. Technol. 52, 291-312 (2006).

5. G. Grossetti et al. DEMO Port Plug design and integration studies // 26th FEC - Kyoto (2016) FIP/7-4.

6. E. Poli et al. TORBEAM, a beam tracing code for electron-cyclotron waves in tokamak plasmas // Comput. Phys. Comm. 136, 90 (2001).

7. S. Garavaglia et al., Preliminary conceptual design of DEMO EC system // AIP Conference Proceedings 1689, 090009 (2015). 